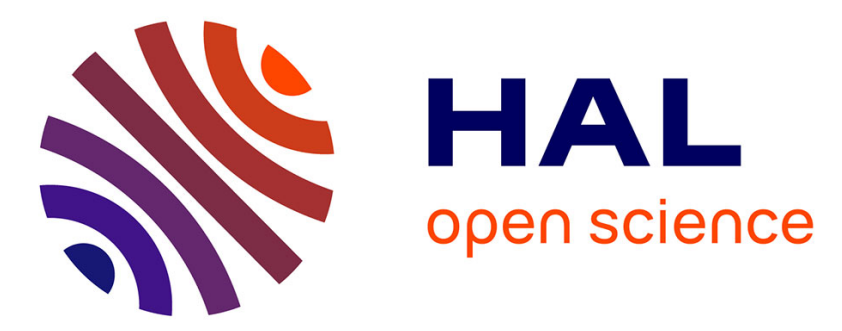

\title{
Prediction of Effective Properties of Porous Carbon Electrodes from a Parametric 3D Random Morphological Model
}

Torben Prill, Dominique Jeulin, François Willot, Juan Balach, Flavio Soldera

\section{- To cite this version:}

Torben Prill, Dominique Jeulin, François Willot, Juan Balach, Flavio Soldera. Prediction of Effective Properties of Porous Carbon Electrodes from a Parametric 3D Random Morphological Model. Transport in Porous Media, 2017, 120 (1), pp.141-165. 10.1007/s11242-017-0913-1 . hal-01678694

\section{HAL Id: hal-01678694 \\ https://hal.science/hal-01678694}

Submitted on 9 Jan 2018

HAL is a multi-disciplinary open access archive for the deposit and dissemination of scientific research documents, whether they are published or not. The documents may come from teaching and research institutions in France or abroad, or from public or private research centers.
L'archive ouverte pluridisciplinaire HAL, est destinée au dépôt et à la diffusion de documents scientifiques de niveau recherche, publiés ou non, émanant des établissements d'enseignement et de recherche français ou étrangers, des laboratoires publics ou privés. 


\section{Prediction of Effective Properties of Porous Carbon Electrodes from a Parametric 3D Random Morphological Model}

Article published in Transport in porous Media 120(1), pp. 141-165. DOI: $10.1007 / \mathrm{s} 11242-017-0913-1$. This is a "post-print" version: minor typographical errors have been corrected (changes highlighted in blue).

Torben Prill • Dominique Jeulin • François Willot · Juan Balach · Flavio Soldera

Received: date / Accepted: date

Torben Prill

Fraunhofer ITWM

Fraunhofer Platz 1

67663 Kaiserslautern

Germany

Tel.: +4963131600 4594

E-mail: prill@itwm.fhg.de

Dominique Jeulin

Center for Mathematical Morphology

MINES Paristech

PSL Research University

Fontainebleau

France

François Willot

Center for Mathematical Morphology

MINES Paristech

PSL Research University

Fontainebleau

France

Juan Balach

Department of Chemistry

Universidad Nacional de Río Cuarto-CONICET

Río Cuarto

Argentina

Flavio Soldera

Department of Materials Science and Engineering

Saarland University

Saarbrücken

Germany 


\begin{abstract}
Pore structures have a major impact on the transport and electrical properties of electrochemical devices, such as batteries and electric double-layer capacitors (EDLCs). In this work we are concerned with the prediction of the electrical conductivity, ion diffusivity and volumetric capacitance of EDLC electrodes, manufactured from hierarchically porous carbons. To investigate the dependence of the effective properties on the pore structures, we use a structurally resolved parametric model of a random medium. Our approach starts from 3D FIB-SEM imaging, combined with automatic segmentation. Then, a random set model is fitted to the segmented structures and the effective transport properties are predicted using full field simulations by iterations of FFT on 3D pore space images and calculations based on the geometric properties of the structure model. A parameter study of the model is used to investigate the sensitivity of the effective conductivity and diffusivity to changes in the model parameters. Finally, we investigate the volumetric capacitance of the EDLC electrodes with a geometric model, make a comparison with experimental measurements and do a parameter study to suggest improved microstructures.
\end{abstract}

Keywords Porous electrodes · Double-layer Capacitor · FIB-SEM nanotomography $\cdot$ Stochastic modeling

\title{
1 Introduction
}

Porous carbon materials are widely used as electrode materials in energy storage devices, such as electrical double-layer capacitors (EDLCs)(Conway, 2013). These devices are used as alternatives or in combination with batteries. Yet, in general, they have a lower specific capacitance per volume and per weight and a higher specific power. In EDLCs, the electric energy is stored in a thin layer on the surface of the porous electrode which makes micro- or nanoporous materials with their large specific surface area especially suitable, since this results in a very high capacitance. Yet, the performance, measure in specific power and capacitance depends on the specific pore shape and size of the electrode materials. Hence, to predict the performance of an electrode material, knowledge and modeling of the morphology of the pore space is necessary, aside from the electrochemical modeling.

To this end, two nanoporous carbon-based materials, used in electrodes of EDLCs, are investigated. The two samples are imaged with FIB-SEM and the images are segmented using a new segmentation algorithm using mathematical morphology as in (Prill et al, 2013). Using the segmented microstructures, a stochastic model is defined for a two-phase heterogeneous material. The first part of the modeling consists of defining a random set model depending on a set of free parameters. Then the best fit parameters are identified by matching the morphological characteristics of the observed microstructures to model realizations. The second part of the paper consists of a model for the effective transport properties of the modeled microstructures. Using the model, the specific conductivity and diffusion resistance of both materials are predicted by simulations using FFT-based methods. Then, we performed a parameter study to estimate the sensitivity of the effective properties with respect to the model parameters. Finally, we use geometric properties of the random set model to predict the volumetric capacitance of the electrodes and make a comparison with measurements. 


\section{State of the Art}

\subsection{Image Analysis and Segmentation}

Reconstruction of highly porous three-dimensional pore spaces from FIB-SEM imaging is still in general an unsolved problem. Different methods have been tried, such as thresholding approaches as in (Nanjundappa et al, 2013) or surface evolution as in (Jørgensen et al, 2010). Other methods include the ones shown in (Salzer et al, 2012), such as threshold backpropagation or valley detection. In this study we use the method presented in (Prill et al, 2013), based on mathematical morphology. Since it has been shown, that even accurately segmented FIB-SEM data can lead to false transport properties, we combine the automatic segmentation with stochastic modeling, as in (Hutzenlaub et al, 2013). Hence, we define a parametric stochastic model, which visually resembles the investigated materials. This allows for a better estimation of the transport propertied as well as a systematic alteration of the model parameters and the optimization of the model with respect to performance measures, such as capacitance and diffusion resistance.

\subsection{Physical Modeling}

Electrochemical double-layer capacitors are devices for the storage of electrical energy by means of an electrical double-layer forming on the surface of the electrode. Physically, energy is stored when charge carriers adsorb to the surface of the electrode and induce a strong electric field across the boundary. This constitutes the so called electrical double layer. The earliest theory of the formation of the double layer was developed by Helmholz in 1879. Later the theory was amended by Stern and then Guy and Chapman. An historical overview can be found in (Conway, 2013). Modern approaches for modeling the double layer include the theories by Bazant, starting with (Bazant et al, 2004).

As the capacitance of the electrode increases with the specific surface area of the electrode, micro- or nanoporous electrodes lend themselves as electrode materials. Different models for porous electrodes for EDLCs have been developed. The simplest ones are based on equivalent circuits, where an overview can be found in (Barsoukov and Macdonald, 2005). A more detailed treatment of the porous electrode has been given in macrohomogeneous models pioneered by de Levie (1963), and extended e.g. in (Paasch et al, 1993) and (Roßberg et al, 1998). A spatially resolving model is given, e.g., in (Wang and Pilon, 2012).

In the present paper we will restrict ourselves to the most basic treatment of the double layer as an areal capacitance and focus on the geometrical aspects of the porous electrode. To this end we are using a stochastic model to represent the morphology of the pore space. The model is based on a Boolean Model of spheres combined with a convolution to achieve a smoothing effect on the geometry.

\section{Materials and Imaging}

The starting point for the study in this paper is segmented FIB-SEM images of two samples of porous carbon electrodes. 


\subsection{Materials}

In this work, there are two samples of different electrode materials under investigation, denoted S12 and S14. Both have been synthesized by the same production process, yet with a slightly different composition, leading to a difference in pore sizes. Both materials have a hierarchical pore space, with mesopores, with a typical pore size of $2 \mathrm{~nm}-50 \mathrm{~nm}$ and micropores, with a typical pore size smaller than $2 \mathrm{~nm}$.

The samples have been produced by carbonization of resorcinol-formaldehyde (RF) gels at $800^{\circ} \mathrm{C}$ in the presence of a cationic surfactant, cetyltrimethylammonium bromide (CTAB), used as a pore stabilizer. As the RF nanoparticles are sintered together during carbonization process, a pore stabilizer is added in order to avoid the collapse of the pores, leading to the formation of a well-connected mesopore network (Balach et al, 2013). The samples S12 and S14 have been produced using a CTAB to resorcinol molar ratio of, respectively, 0.12 and 0.14 , leading to a difference in the morphology of the mesopore network. Based on (Balach et al, 2013), in this study it is assumed that the micropore morphology is the same for both samples.

\subsection{Imaging and Segmentation}

The two samples were imaged by FIB-SEM Nanotomography (Balach et al, 2012), using the secondary electron signal. The resolution of the SEM image stack of S12 was $3.57 \mathrm{~nm} \times 3.57 \mathrm{~nm}$ and the slicing with the focused ion beam was carried out with $10 \mathrm{~nm}$ thickness. Additionally, the tilting of the sample by $52^{\circ}$ leads to a larger resolution in $y$-direction. This leads to a resulting voxel size in the threedimensional image of $3.57 \mathrm{~nm} \times 3.62 \mathrm{~nm} \times 10 \mathrm{~nm}$. A subwindow of the dimension $411 \times 311 \times 181$ voxels was cropped from the original image stack for the analysis. To improve the segmentation result, the image was magnified by a factor of 1.5 yielding a data set of dimension $616 \times 496 \times 271$ voxels with a voxel size of $2.38 \mathrm{~nm}$ $\times 2.41 \mathrm{~nm} \times 6.67 \mathrm{~nm}$.

The sample S14 was imaged with a lateral resolution of $5 \mathrm{~nm} \times 5 \mathrm{~nm}$, with a $10 \mathrm{~nm}$ slicing thickness, leading to a voxel size of $5 \mathrm{~nm} \times 6.27 \mathrm{~nm} \times 10 \mathrm{~nm}$. Analogously to S12, a subwindow of dimension $611 \times 293 \times 123$ voxels was cropped from the image stack. Fig. 1 shows two slice views of the cropped FIB-SEM image stack of S12 (on the left) and S14 (right).

After imaging and cropping, the images stacks were segmented using the morphological algorithm presented in (Prill et al, 2013), which uses the shading effect present in the SEM images, as the result of the large depth of field of the instrument. The segmentation parameters were chosen manually to optimize the visual impression of the segmentation. Slice views through the segmented images are shown in Fig. 2. The morphology of the porous network shows two tortuous components of a bipercolating medium, requiring the choice of adequate random textures models to generate a faithful description.

Finally, the images were scaled to yield an isotropic voxel edge length of $2.38 \mathrm{~nm}$ for $\mathrm{S} 12$ and $5 \mathrm{~nm}$ for S14. 


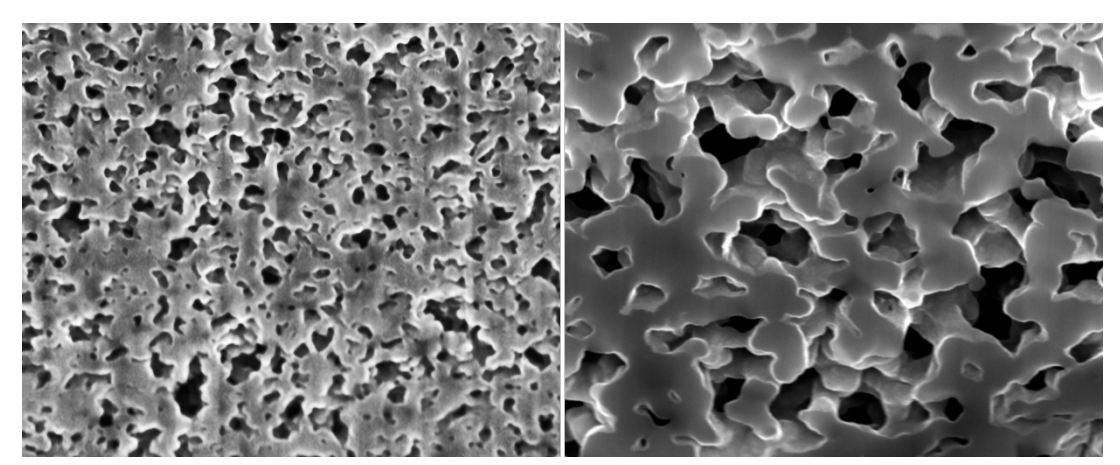

Fig. 1 Slice views of the FIB-SEM image stacks for the samples S12 (left) and S14 (right).

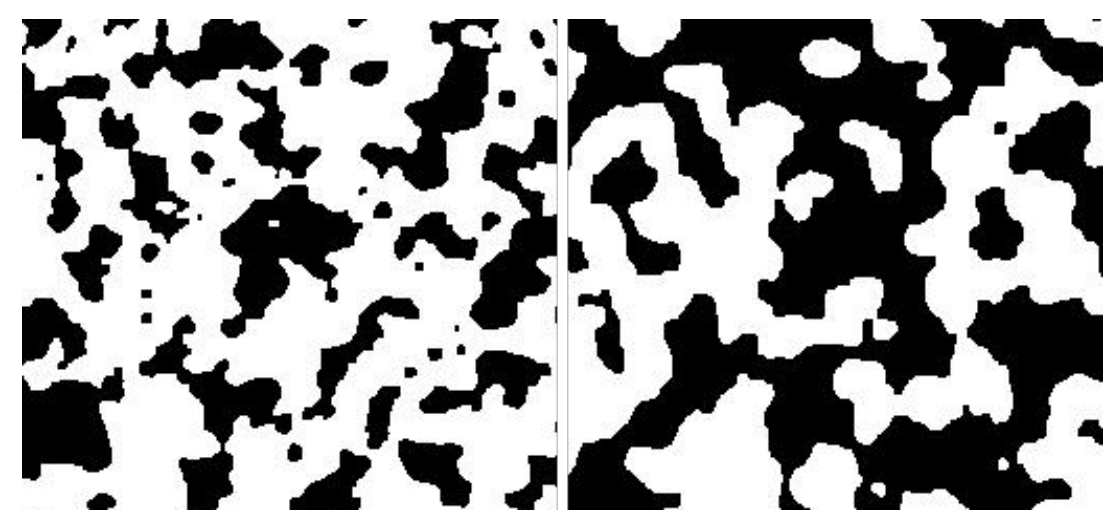

Fig. 2 Slice views of the segmentations of the images of samples S12 (left) and S14 (right).

\section{Modeling the Morphology Nanoporous Carbon Electrodes}

Since it has been shown, that computations of transport properties based on segmented FIB-SEM images can lead to large errors in the estimated properties resulting from the lack of spatial resolution in the $z$ direction (Hutzenlaub et al, 2013 ), it was decided to use a model of random set for the generation of 3D computational domains used as input data for estimation of the transport properties. This allows for correcting errors in the segmentation by, e.g., modeling an isotropic structure, and to explore systematic modifications of the microstructure. Starting from the segmented images, morphological measurements were made to estimate some probabilistic properties of the samples. This is the first step to generate representative domains matching the material morphology as best as possible. The random set model depends on a set of free parameters, which have to be determined by model fitting. Since both samples are fitted to the same model, this results in two sets of parameters representing the respective microstructures of the samples. It opens the possibility to make a systematic study of the impact of the morphology of the mesopores on the transport properties of electrodes. 
4.1 Principle of Random Set Modeling

A random set $A$ is completely known and identified from a functional, its Choquet capacity, defined on compact sets $K$ in (Matheron, 1967), (Matheron, 1975; Serra, 1982; Jeulin, 2000) defined by

$$
T(K)=P(K \cap A \neq \varnothing)
$$

where $P$ is a probability. If we denote $K_{x}$ the compact set $K$ after translation to point $x$, we get

$$
T\left(K_{x}\right)=P\left(K_{x} \cap A \neq \varnothing\right)=P\{x \in A \oplus \check{K}\}
$$

where $A \oplus \check{K}$ is the result of the dilation of set $A$ by $K$ :

$$
A \oplus \check{K}=\left\{x, K_{x} \cap A \neq \emptyset\right\}=\cup_{y \in K} A_{-y}=\cup_{x \in A, y \in K}\{x-y\}
$$

Similarly, we can define the erosion by $K, A \ominus \check{K}$, from

$$
A \ominus \check{K}=\left\{x, K_{x} \subset A\right\}=\cap_{y \in K} A_{-y}=\left(A^{c} \oplus \check{K}\right)^{c}
$$

where $A^{c}$ is the complementary set of $A$. In principle, all types of compact sets should be used for a complete characterization of $A$. In what follows, we will use pair of points $(K=\{x, x+h\})$ and approximation of spheres in 3D by rhombo-cuboctaedra.

In addition to the Choquet capacity, size distributions of a random set can be accessed through the use of the opening transformation by convex compact sets (like a sphere with radius $r, B(r)$ ). This transformation starts from an erosion of the set by $B(r)$, and is followed by a dilation by $B(r)$ :

$$
\gamma_{B(r)}(A)=(A \ominus B(r)) \oplus B(r)
$$

Alternatively, the size distribution of $A^{c}$ is accessed from the closing transformation of $A$ by convex sets. For spheres $B(r)$ the transformation starts from a dilation of $A$ by $B(r)$ and is followed by an erosion by $B(r)$ :

$$
\phi_{B(r)}(A)=(A \oplus B(r)) \ominus B(r)
$$

It turns out that opening operations with spheres of increasing radius $r$ progressively removes details of the random set $A$ until its complete suppression. Voxels disappeared for size $r$ correspond to details in $A$ with size less than $r$, so that a cumulative size distribution can be easily obtained from the estimation of the probability for a point $x$ to belong to $\gamma_{B(r)}(A)$, obtained by its volume fraction. Similarly, closing operations by spheres of increasing radius progressively fill the space, and removes details of $A^{c}$, giving access to a cumulative size distribution of $A^{c}$, estimated from the measurement of the volume fraction of $\phi_{B(r)}(A)$. Applied to the segmented binary images of the nanoporous carbon electrodes of this study, both size distributions of the carbon phase and of the mesopores are estimated in $3 \mathrm{D}$.

A typical size of $A$, namely a median radius $r_{M}$, is obtained for

$$
P\left\{x \in \gamma_{B\left(r_{M}\right)}(A)\right\} / P\{x \in A\}=0.5
$$


. Similarly, a typical size of $A^{c}$, is obtained for

$$
P\left\{x \in \phi_{B\left(r_{M}\right)}(A)\right\}-P\{x \in A\} /(1-P\{x \in A\})=0.5
$$

The same operations and measurements are implemented on the 3D images of materials and of 3D realizations of parametric random sets in an iterative process aiming to minimize some distance between real and virtual specimens, as illustrated later.

\subsection{Random Set Model}

As a starting point for the modeling we use a Boolean model (Matheron, 1967, 1975; Serra, 1982; Jeulin, 2000). It is built in two steps: a Poisson point process with intensity parameter $\theta$ (average number of points per unit volume) generates random germs in the $3 \mathrm{D}$ space; independent realizations of a random primary grains $A^{\prime}$ are located on the Poisson points $x_{k}$. The random set $A$ is obtained by the union of $A_{x_{k}}^{\prime}$ :

$$
A=\bigcup_{k} A_{x_{k}}^{\prime}
$$

For this model the Choquet capacity is known in closed form. In the $3 \mathrm{D}$ space, we have, denoting $\bar{V}$ the mathematical expectation of the volume:

$$
T(K)=1-\exp \left(-\theta \bar{V}\left(A^{\prime} \oplus \check{K}\right)\right.
$$

In the present case, we can use a monodisperse Boolean Model of Spheres (BSM) for the mesopores, the primary grain being a sphere of radius $r, B(r)$. It turns out that for a volume fraction of spheres larger than 0.3 and less than 0.95 , this model is bipercolating (Jeulin and Moreaud, 2005), which is required for the present materials.

A slice view through a realization of the Boolean model used for the modeling is shown in Fig. 3a.

Yet, clearly the segmented sample images do not resemble the morphology of the Boolean model, as the union of two spheres produces corners, which are not present in the segmented images. Hence, it was decided to modify the BSM by smoothing its boundary. This is done by using the indicator function of the BSM defined as

$$
\mathbf{1}_{A}(x)=\left\{\begin{array}{lll}
1 & \text { if } & x \in A \\
0 & \text { if } & x \in A^{c}
\end{array} .\right.
$$

To generate realizations of the modified model, the indicator function is convolved by a centered Gaussian kernel with width $\sigma$ and integral 1 over the 3D space,

$$
N(0, \sigma)(x)=\frac{1}{\sqrt{2 \pi \sigma^{2}}} e^{-\frac{x^{2}}{2 \sigma^{2}}} .
$$

This yields a random function $Z(x)$, with values in the interval $[0,1]$ : 


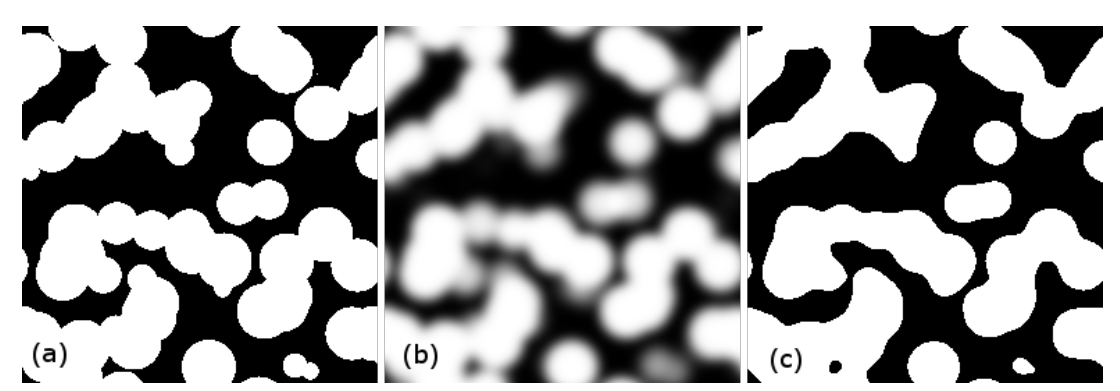

Fig. 3 Realizations of a Boolean model of spheres (a), a smoothed indicator function $Z(x)$ of the BSM (b) and a random set $A_{1}$ obtained by thresholding $Z(x)$ (c).

$$
Z(x)=\mathbf{1}_{A} * N(0, \sigma) .
$$

A realization of $Z(x)$ is shown on Fig. 3b. To obtain the indicator function of a random set $A_{1}$, a threshold $C$ is applied, as shown on Fig. 3c:

$$
A_{1}=\{x, Z(x) \geq C\}
$$

The "convolution-thresholding" approach (Grzhibovskis and Heintz, 2005) gives a good approximation of the evolution of the boundaries of the initial random set $A$ by motion with a local velocity proportional to the local mean curvature in the present case. It has the effect to smoothen the irregularities of the boundary, as would be the case for some reaction-diffusion process.

In the present case, this sequence yields a random set depending on four parameters $\theta, r, \sigma$ and $C$.

The intensity $\theta$ can vary between zero and infinity but shows very small changes, when the volume fraction is close to zero or to unity. This hampers the optimization involved in the identification of parameters, which is based in a gradient descent. Hence, the parameter $\theta$ is replaced by the volume fraction of the BSM $p$ using the relation derived from Eq. 2:

$$
p=1-\exp \left(-\theta \frac{4}{3} \pi r^{3}\right)
$$

The parameter $p$ varies only between zero and unity and is hence more stable during the identification. To further simplify the fitting, the threshold $C$ is chosen such that the volume fraction of the set $A_{1}$ is equal to the volume fraction of the BSM on each realization. This can be achieved by analyzing the histogram of realizations of the random function $Z(x)$. This eliminates $C$ as a free parameter, the parameter $p$ being directly estimated from the volume fraction of segmented images.

\subsection{Fitting Free Parameters to Images}

To estimate the free model parameters giving the best match to the segmented images, a distance was defined, in order to measure the morphological similarity between model realizations and the segmented images. The distance $Y$ is the 
sum of the squared difference of three morphological measurements (Serra, 1982) performed on the segmented images and on realizations of the model. These morphological measurements are the set covariance, the opening curve and the closing curve.

\subsubsection{Set Covariance}

The set covariance is given by the probability of two points, separated by a distance $h$, to be included in the random set $A$ :

$$
\operatorname{Cov}(h)=P(x \in A, x+h \in A)
$$

For a stationary random set with finite scale, the covariance reaches its sill $(\operatorname{Cov}(0))^{2}$ for a finite length $a$, called its range. For the studied materials, the covariance is invariant by rotation of vector $h$, so that their morphology is isotropic in $3 \mathrm{D}$.

\subsubsection{Opening curve}

The opening curve $\Gamma(r)$ is given by the volume fraction $V_{V}$ of the random set after a morphological opening by the spheres $B(r)$

$$
\Gamma(r)=V_{V}\left[\gamma_{B(r)}(A)\right]
$$

\subsubsection{Closing Curve}

Analogously, the closing curve $\Phi(r)$ is defined by the volume fraction of the random set after closing by the spheres $B(r)$

$$
\Phi(r)=V_{V}\left[\phi_{B(r)}(A)\right]
$$

For the identification, these functions were sampled at several distances $h_{i}$ and radii $r_{i}$ yielding the distance $Y$, defined as (the subscripts $d$ and $m$ stating for experimental data and for simulated model)

$$
\begin{aligned}
Y & =\sum_{h_{i}}\left[\operatorname{Cov}_{d}\left(h_{i}\right)-\operatorname{Cov}_{m}\left(h_{i}\right)\right]^{2} \\
& +\sum_{l_{i}}\left[\Gamma_{d}\left(r_{i}\right)-\Gamma_{m}\left(r_{i}\right)\right]^{2} \\
& +\sum_{l_{i}}\left[\Phi_{d}\left(r_{i}\right)-\Phi_{m}\left(r_{i}\right)\right]^{2}
\end{aligned}
$$

The covariance was sampled on 37 equidistant points, spanning the interval $h_{i}=$ $[0,76]$ voxels. Each of the other two curves were sampled on 15 points covering the radii $[0,30]$ voxels. This gives the covariance roughly twice the weight of the other data.

It is assumed that the best parameter fit is reached for a minimum of the distance $Y$. Since, the measurements on the model are made on realizations, $Y$ is prone to noise, and therefore a stochastic minimization algorithm was used, the SPSA algorithm in (Spall, 1992) in the present study. 
The algorithm starts with a given set of parameters

$$
\theta_{0}=\left(r_{0}, \sigma_{0}\right) .
$$

Then a random sequence of computed according to the update rule

$$
\theta_{k+1}=\theta_{k}-a_{k} g_{k}\left(\theta_{k}\right),
$$

with the stochastic gradient

$$
g_{k}\left(\theta_{k}\right)=\left(\begin{array}{c}
\frac{Y\left(\theta_{k}+c_{k} \Delta_{k}\right)-Y\left(\theta_{k}-c_{k} \Delta_{k}\right)}{2 c_{k} \Delta_{k, 1}} \\
\vdots \\
\frac{Y\left(\theta_{k}+c_{k} \Delta_{k}\right)-Y\left(\theta_{k}-c_{k} \Delta_{k}\right)}{2 c_{k} \Delta_{k, i}}
\end{array}\right) .
$$

The sequences $\left(a_{k}\right)$ and $\left(c_{k}\right)$ control the convergence of the sequence, while the sequence $\left(\Delta_{k}\right)$ leads to a perturbation in parameter space, with the components of $\left(\Delta_{k}\right)$ following a Bernoulli distribution, i.e., $P\left(\Delta_{k, i}= \pm 1\right)=0.5$.

In the present study, the sequences

$$
\begin{aligned}
& a_{k}=\frac{a}{(k+1)^{\alpha}}, a=0.1, \alpha=0.101 \\
& c_{k}=\frac{c}{(k+1)^{\gamma}}, c=0.03, \gamma=0.602
\end{aligned}
$$

have been used. This, leads to a slow but steady convergence to the minimum of the objective function $Y(\theta)$.

\subsection{Experimental Results}

The main 3D morphological properties are summarized in Table 1, clearly showing the differences in the scales of the two mesostructures.

\subsection{Optimal Model Fit}

For the model fitting, the stochastic optimization method presented in (Spall, 1992) was performed with the distance given in Eq. 11. The realizations of the modified BSM described in Sect. 4.2 for the fitting were generated on a voxel grid with dimension $256 \times 256 \times 256$. The stochastic optimization converged to a minimum for each of the samples, leading to the respective best fit parameters. Overall, the model fitting yielded the following results.

Table 1 Basic morphological properties of the two samples: solid volume fraction $(p)$; range of covariance $\left(r_{c}\right)$; median radius solid $\left(\operatorname{med}\left(r_{s}\right)\right) ;$ median pore radius $\left(\operatorname{med}\left(r_{p}\right)\right)$

\begin{tabular}{lcccc}
\hline Sample & $p$ & $r_{c}$ & $\operatorname{med}\left(r_{s}\right)$ & $\operatorname{med}\left(r_{p}\right)$ \\
\hline S12 & $67 \%$ & $47.6 \mathrm{~nm}$ & $23.8 \mathrm{~nm}$ & $14.28 \mathrm{~nm}$ \\
S14 & $52 \%$ & $150 \mathrm{~nm}$ & $50 \mathrm{~nm}$ & $60 \mathrm{~nm}$
\end{tabular}


Table 2 Optimal parameters obtained by fitting to the observed structure of S12

\begin{tabular}{lcc}
\hline Parameter & Optimal value (voxel) & Optimal value (nm) \\
\hline$p$ & 0.67 & 0.67 \\
$r$ & 7 & 16.7 \\
$\sigma$ & 10 & 23.8 \\
\hline
\end{tabular}

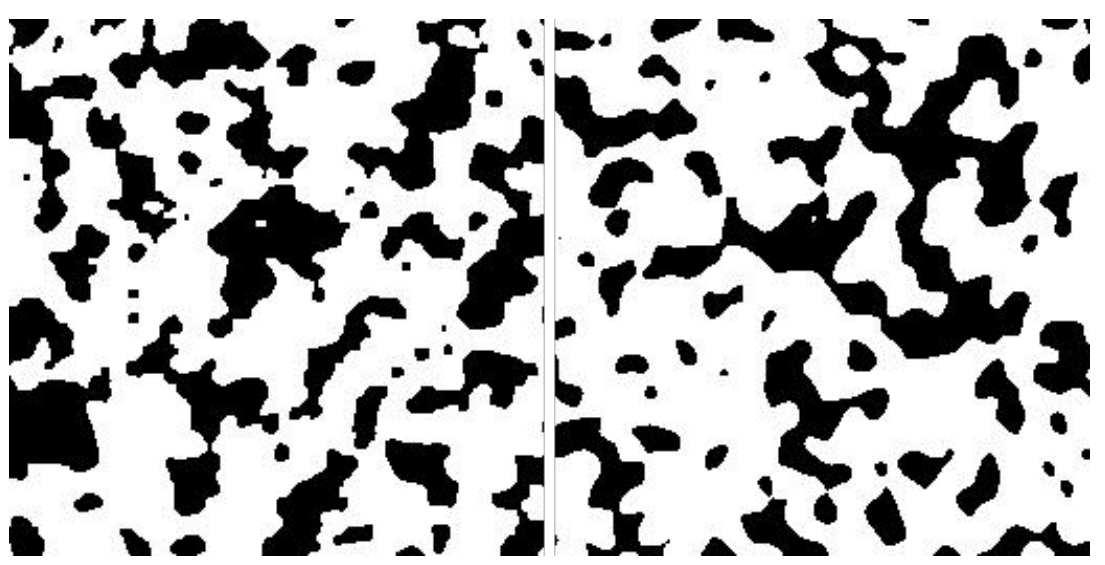

Fig. 4 Comparison between the observed structure (left) and the fitted model (right) of dataset S12.

\subsubsection{Fitting S12}

Optimal parameters for a model representing the sample S12 are shown in Table 2 .

A comparison between the segmented image and a microstructure realization is shown in Fig. 4. As can be seen, the model reproduces the shape of the pores and the solid phase quite well. A slight difference can be observed in that the boundary of the model is slightly coarser, yet this might be due to noise induced by errors in the segmentation.

To quantify the similarity, the curves for the morphological measurements on the segmentation and on the model realizations are shown in Figs 5-7. As shown in Fig. 5 the covariance of the model realization is in good agreement with the one measured on the segmented image. Also, the opening curves in Fig. 6 are in good agreement. Yet, a small deviation can be observed in that the opening curve on the model shows a slightly lower probability to find larger details in the solid phase. The closing curves of the model and the segmentation (Fig. 7) virtually coincide, meaning that the size distribution of pores is recovered in the simulations of the model.

\subsubsection{Fitting S14}

Applying the same fitting procedure to the micrograph of sample S14, results in the parameters given in Tab. 3. A visual comparison of model and segmented image is shown in Fig. 8. 


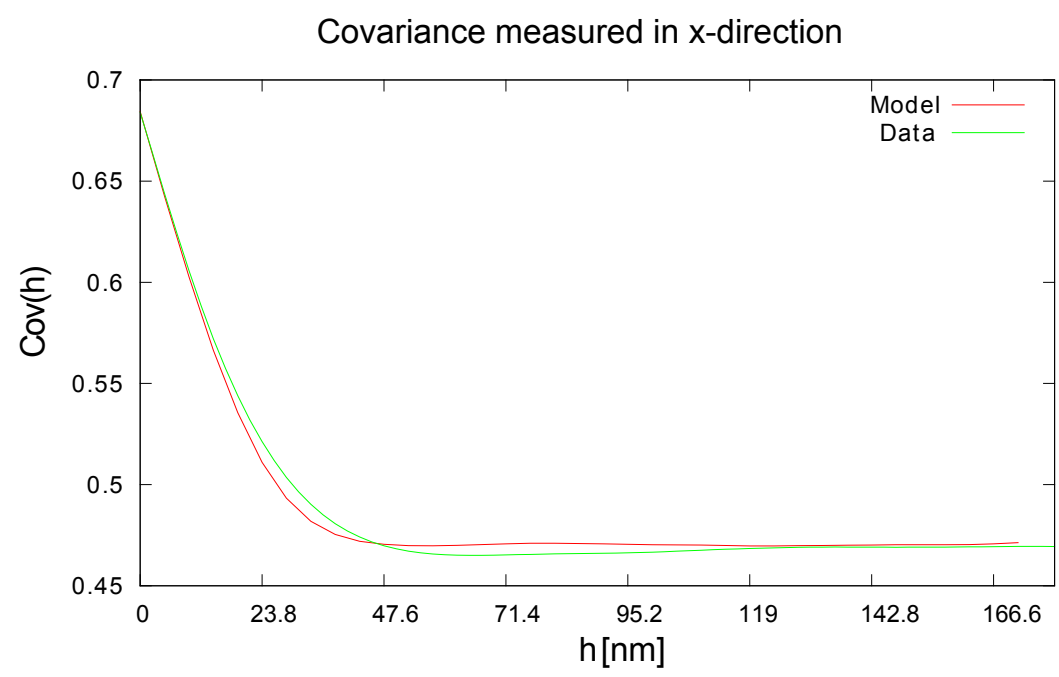

Fig. 5 Comparison between the covariance of the fitted model (red) and the observed structure (green) of dataset S12.

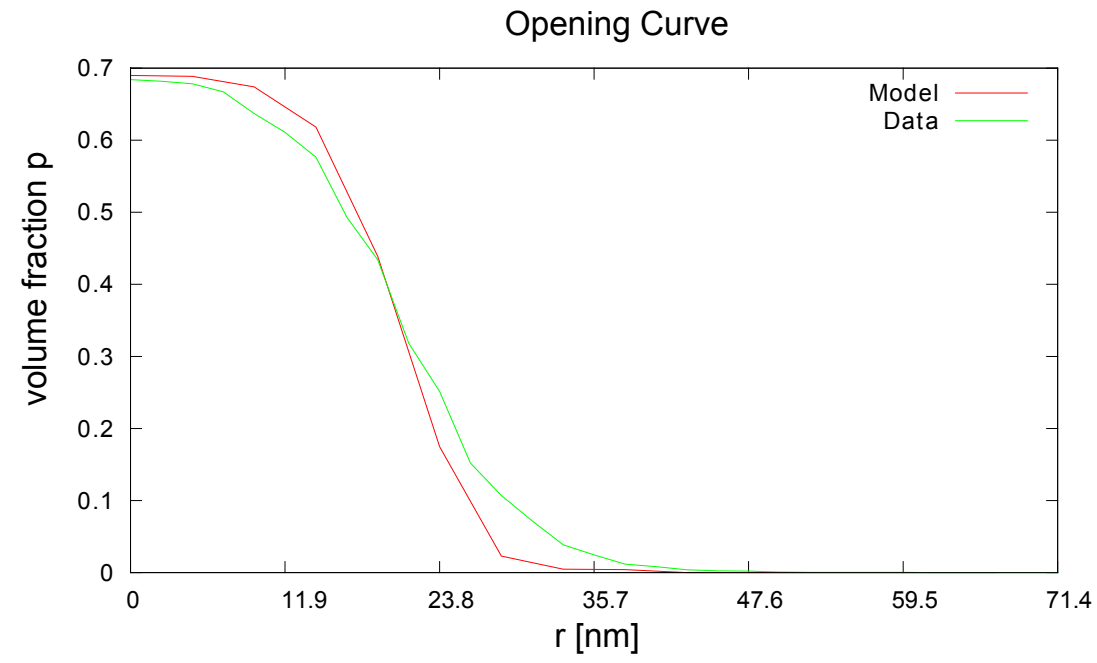

Fig. 6 Comparison between the opening curve of the fitted model (red) and the observed structure (green) of dataset S12.

Table 3 Optimal parameters obtained by fitting to the observed structure of S14

\begin{tabular}{lcc}
\hline Parameter & Optimal value (voxel) & Optimal value $(\mathrm{nm})$ \\
\hline$p$ & 0.52 & 0.52 \\
$r$ & 10 & 50 \\
$\sigma$ & 14 & 70 \\
\hline
\end{tabular}




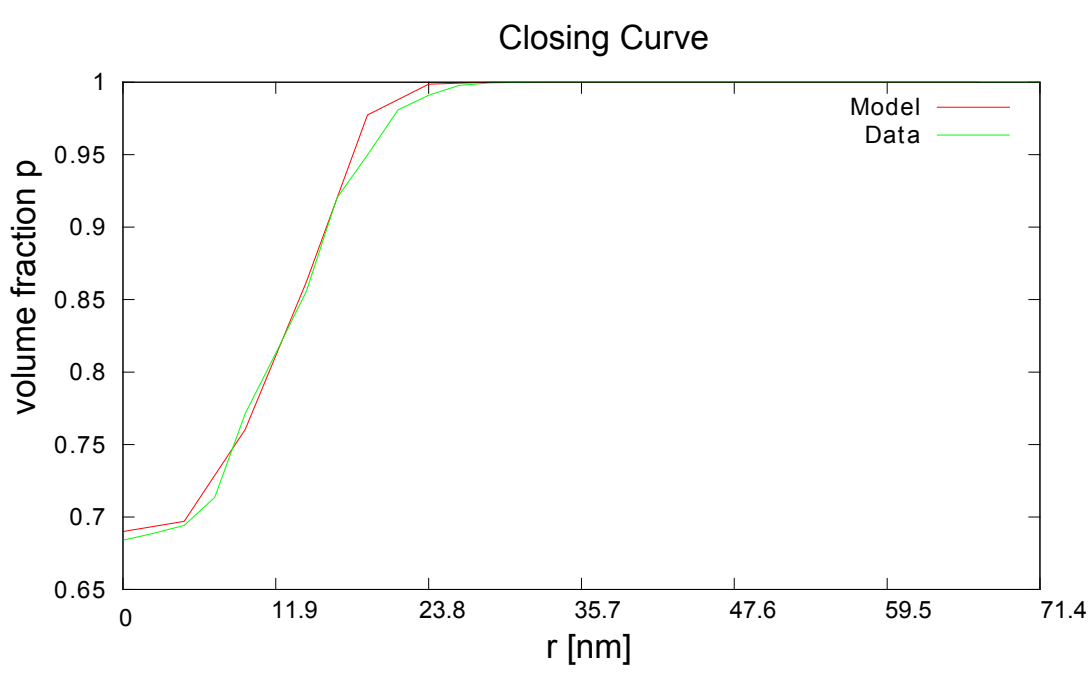

Fig. 7 Comparison between the closing curve of the fitted model (red) and the observed structure (green) of dataset S12.

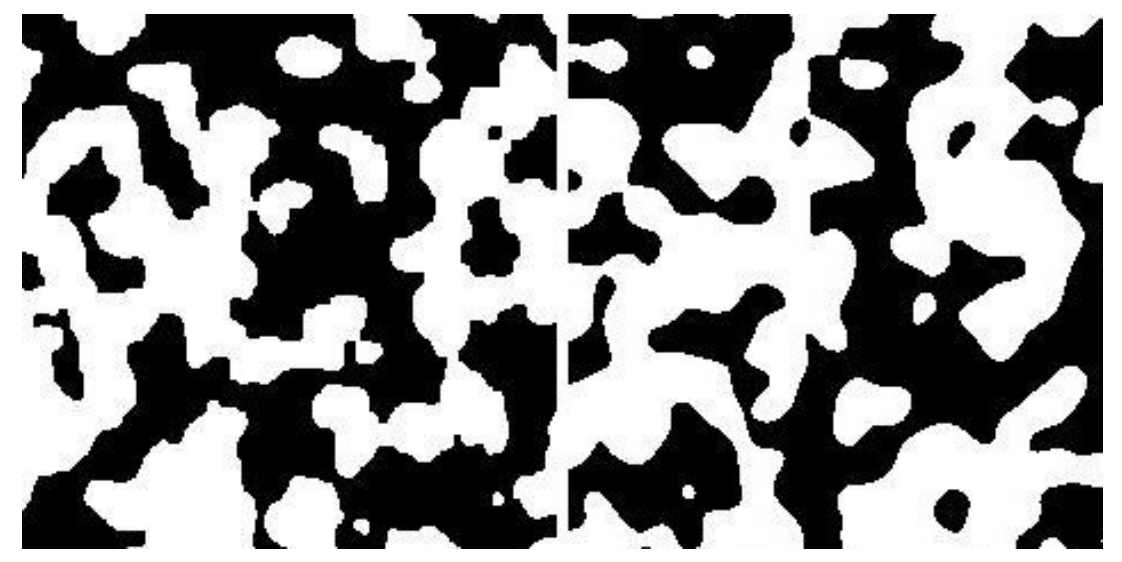

Fig. 8 Comparison between the observed structure (left) and the fitted model (right) of dataset S14.

The fitted model to S14 shows even better agreement in the measured characteristics. The covariances plotted in Fig. 9 and the opening curves plotted in Fig. 10 coincide almost perfectly, while a small deviation can be observed in the closing curves (Fig. 11), this time the model tending to less larger pore sizes.

Overall, as previously mentioned, the microstructure of S14 is much coarser than the S12s, as the fitted radii of the initial spheres as well as the width of the filter mask is about a factor $2-3$ times larger. Although the volume fraction is larger in S12, the amount of coarsening necessary to reproduce the microstructure for both samples is similar. This is indicated by the fact that the ratio between the initial radii and the width of the filter mask is about $\frac{\sigma}{r}=1.4$, for both materials. 


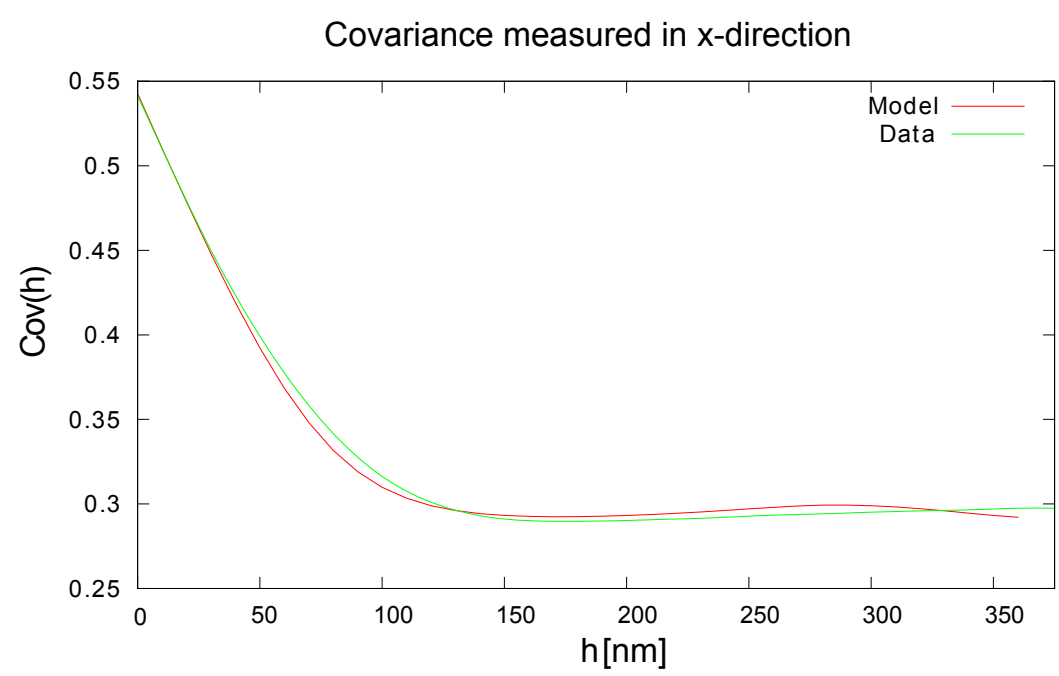

Fig. 9 Comparison between the covariance of the fitted model (red) and the observed structure (green) of dataset S14.

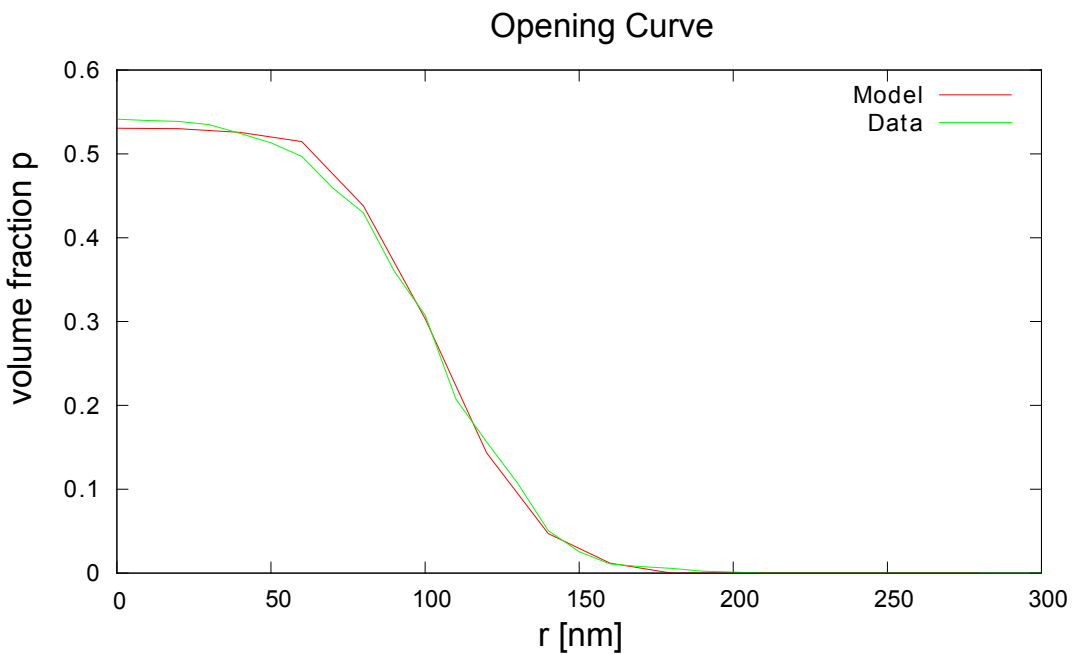

Fig. 10 Comparison between the opening curve of the fitted model (red) and the observed structure (green) of dataset S14.

Finally, the surface area of both samples has been measured on the segmented images, as well as on the modeled microstructures. This is an important feature since the surface area of the mesopores plays an important role in capacitance of the materials. The resulting values are shown in Table 4. As can be seen, the values differ by up to $18 \%$ for the sample S14. This discrepancy is attributed to errors in the segmentation, since it is known that the segmentation algorithm does not reconstruct the surface of objects perfectly. 


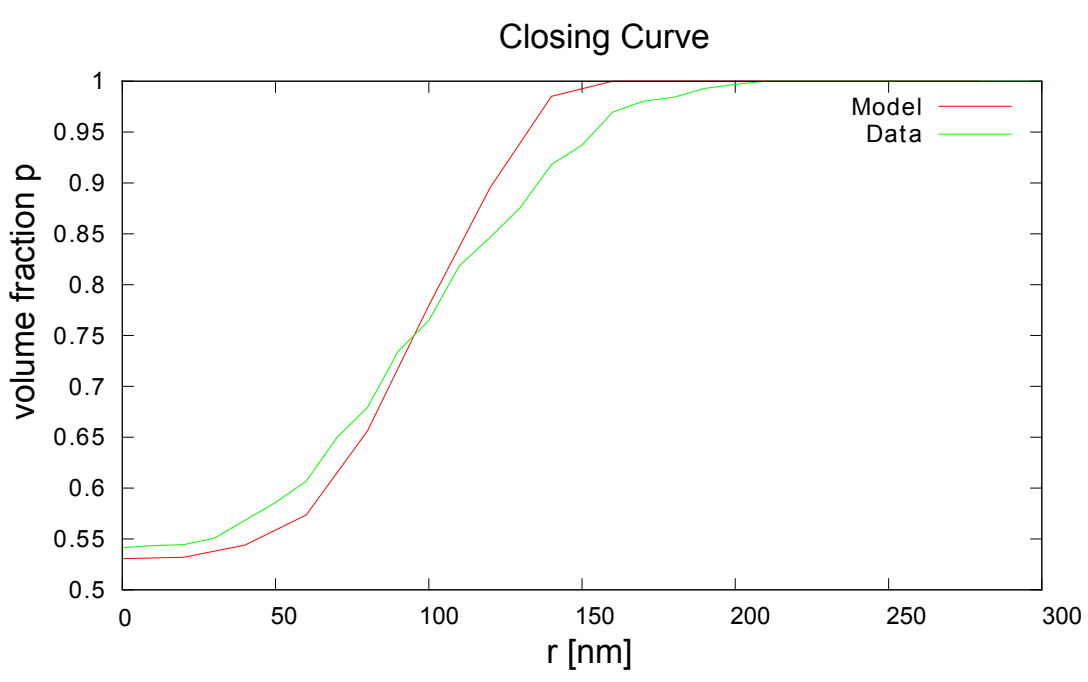

Fig. 11 Comparison between the closing curve of the fitted model (red) and the observed structure (green) of dataset S14.

\section{Estimating the Effective Transport Properties}

\subsection{Method of Numerical Homogenization}

In order to predict the transport properties of the different mesopore structures, the effective conductivity and the effective diffusivity of the samples were estimated by numerical homogenization (Papanicolau et al, 1978; Sánchez-Palencia, 1980), starting from model realizations of the modified BSM described in Sect. 4.2. In this study, the porous material is modeled as a two-phase heterogeneous material as shown in Fig. 12. The main difficulty concerns the contrast between the properties of components, which is infinite in the present case. Therefore, other estimation techniques like bounds derived from variational techniques can only provide an upper bound, the lower bound being equal to zero.

It is assumed that the local material properties are given by a constant conductivity $\sigma_{1}$ in the solid phase and a zero conductivity in the pore space. To estimate the effective conductivity of the material, we have to compute the electric field $E(x)$ deriving from the potential $\Phi(x)$ in realizations of the medium, by solving

Table 4 Specific surface area measurement on the segmented images and the modeled structures.

\begin{tabular}{lcc}
\hline Sample & $a_{\text {seg }}\left[\frac{\mathrm{cm}^{2}}{\mathrm{~cm}^{3}}\right]$ & $a_{\bmod }\left[\frac{\mathrm{cm}^{2}}{\mathrm{~cm}^{3}}\right]$ \\
\hline S12 & $3.1 \times 10^{5}$ & $3.28 \times 10^{5}$ \\
S14 & $7.15 \times 10^{4}$ & $6.02 \times 10^{4}$ \\
\hline
\end{tabular}




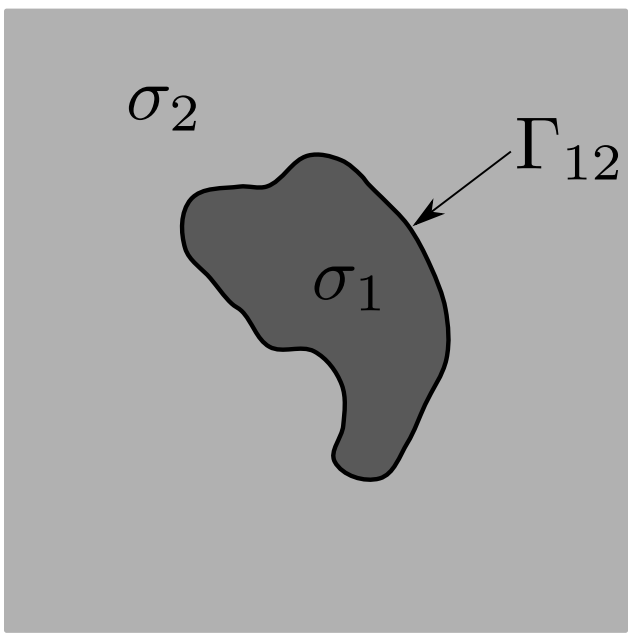

Fig. 12 A sample domain on which the effective conductivity and diffusivity are computed.

the problem

$$
\begin{aligned}
\nabla \cdot[\sigma(x) \nabla \Phi(x)] & =0, \\
\nabla \times E(x) & =0,
\end{aligned}
$$

with periodic boundary conditions on the domain boundaries and the condition $\langle E(x)\rangle=\bar{E},\langle E(x)\rangle$ meaning the space average of the electric field. The problem is numerically solved using the "accelerated" Fourier scheme (Eyre and Milton, 1999). It is an efficient method, which directly applies to images and does not require any meshing of the microstructure. The method is based on rewriting Eq. 16 as the implicit integral equation

$$
E(x)=\bar{E}-\int \Gamma\left(x^{\prime}\right) P\left(x-x^{\prime}\right) d x^{\prime},
$$

where $\Gamma$ is the (second-rank, periodic) Green operator associated to the homogeneous conductivity $\sigma_{0}$ (Duffy, 2001) and $P(x)=J(x)-\sigma_{0} E(x)$ is the polarization field associated to $\sigma_{0}$.

In the accelerated FFT method, (18) is solved by a Neumann series, which is computed by an iterative fixed-point algorithm. Using explicit formula for the Green operator, the convolution product in the right-hand-side of (18) is determined in the Fourier domain. The use of fast Fourier transforms greatly reduces computation times. Additionally, for the Green operator, use is made of the "finitedifference" discretization proposed in (Willot et al, 2014), which improves the convergence rate and also leads to more precise local fields. In FFT algorithms, the convergence rate generally depends on the reference conductivity $\sigma_{0}$. In the present method, its optimal value is unknown. Based on numerical experiments, we set $\left|\frac{\sigma_{0}}{\sigma_{1}}\right|=0.36$ for all computations. We stress that this value is not necessarily optimal.

The effective conductivity $\sigma_{\text {eff }}$ is estimated using:

$$
\sigma_{\text {eff }}\left\langle E_{i}(x)\right\rangle=\left\langle\sigma(x) E_{i}(x)\right\rangle=\left\langle J_{i}(x)\right\rangle,
$$


where a macroscopic potential gradient $\langle\nabla \phi(x)\rangle$ is applied along the direction $\mathrm{e}_{i}$.

The diffusivity is estimated by the same approach, since we consider a steady state for the diffusion, so that the time derivative of the concentration is equal to zero. For this problem, the diffusivity of the solid phase is equal to zero, while the diffusivity of pores, filled with some electrolyte, is set to one. Therefore, the conductivity problem and the diffusivity problems are solved on the same mesostructure, after exchanging the roles of pores and of the solid phase.

\subsection{Predicting Effective Transport Properties}

\subsubsection{Bounds of effective properties}

As a first estimate for the effective transport properties, the Wiener, HashinShtrikman, and third-order upper bounds are computed for the effective conductivity as well as the effective diffusivity. Since one component has a vanishing transport property, the lower Wiener, Hashin-Shtrikman and third-order bounds vanish.

The upper Wiener bound is valid for any microstructure of a heterogeneous material, while the upper Hashin-Shtrikman bound are valid for isotropic microstructures. Both depend only on the volume fractions of the phases $p_{1}$ and $p_{2}$ and the bulk properties of the respective phases, $\sigma_{1}$ and $\sigma_{2}$. The upper Wiener bounds for a two-phase material with vanishing property for $\sigma_{2}$ are given by

$$
\sigma_{W}^{u}=p_{1} \sigma_{1}
$$

For a two-phase material, the upper Hashin-Shtrikman bounds are given by:

$$
\sigma_{\mathrm{HS}}^{u}=\sigma_{1}\left(1+\frac{p_{2}}{1+\frac{p_{2}}{3}}\right) .
$$

The third-order bounds (Beran, 1965) make use of the 3-points probability functions $P\left\{x \in A, x+h_{1} \in A, x+h_{2}\right\}$ and are generally model-dependent. For practical applications to two-components media with an isotropic geometry, they depend separately on a function calculated by some integral of the 3-points probability and on the property of each component (Milton, 1982). This probability is not known in a closed form for the present "convolution-thresholding" model, but it can be approximated by the corresponding function for the BSM model:

$$
\begin{aligned}
& P\left\{x \in A, x+h_{1} \in A, x+h_{2}\right\}= \\
& \quad \exp \left(-\theta V\left(B(r) \cup B(r)_{-h_{1}} \cup B(r)_{-h_{2}}\right)\right)
\end{aligned}
$$

This expression was used to estimate the function involved in the calculation of the third-order upper and lower bounds $\sigma_{\mathrm{BSM}}^{u}$ and $\sigma_{\mathrm{BSM}}^{l}$ of the BSM (Torquato and Stell, 1985), which depends linearly on the volume fraction of spheres $p$. Many other models of random media show a similar behavior (Jeulin, 2005).

For the upper Wiener, Hashin Shtrikman, and third order bounds for the effective conductivity and diffusivity of the modeled microstructures are shown in Tables 5 and 6 , respectively. 
Table 5 Upper Wiener, Hashin-Shtrikman and third-order bounds of the conductivity of the two samples

\begin{tabular}{lccc}
\hline sample & $\sigma_{\mathrm{W}}^{u}$ & $\sigma_{\mathrm{HS}}^{u}$ & $\sigma_{\mathrm{BSM}}^{u}$ \\
\hline $\mathrm{S} 12$ & 0.67 & 0.629 & 0.557 \\
$\mathrm{~S} 14$ & 0.52 & 0.428 & 0.3914 \\
\hline
\end{tabular}

Table 6 Upper Wiener, Hashin-Shtrikman and third-order bounds of the diffusivity of the two samples

\begin{tabular}{lccc}
\hline sample & $D_{\mathrm{W}}^{u}$ & $D_{\mathrm{HS}}^{u}$ & $D_{\mathrm{BSM}}^{u}$ \\
\hline $\mathrm{S} 12$ & 0.33 & 0.137 & 0.117 \\
$\mathrm{~S} 14$ & 0.48 & 0.37 & 0.244 \\
\hline
\end{tabular}

\subsubsection{Effective Conductivity}

The effective conductivities were predicted by solving the problem in Eqs. 16-19 with the software morphhom (Willot et al, 2014). Hence, the conductivities are computed for the electrostatic case (i.e., at frequency $\omega=0$ ). This is justified, by the low frequency the capacitances being measured in the EIS measurement.

For the determination of the effective conductivities, 20 model realizations with different dimensions between $128^{3}-512^{3}$ voxels were generated for each of the parameter sets in Tables 2 and 3 . Then, the effective conductivities were computed for each realization. From the statistical dispersion of conductivities, it is possible to estimate the representativeness of the computed values with respect to variations in the model realizations. A study on the statistical representative volume element (as defined in Kanit et al (2003)) for S12's model is shown in Fig. 13. To characterize the statistical dispersion of the conductivity, the mean and the empirical standard deviation were computed for the 20 realizations of the model for each edge length of the volume. Fig. 13 shows the mean and twice the standard deviation of the effective conductivity as error bars. Hence, the error bars correspond to the $95 \%$ confidence interval of the individual realizations. As can be seen, the realizations with edge length greater than 256 voxel, corresponding to a physical volume with edge length of about $l \approx 0.6 \mu \mathrm{m}$, show no bias (there is no edge effect for this size) and have a relative precision of less than $\pm 2 \%$.

When combining the 20 realizations with the largest edge length of 512 voxels, the confidence interval of the effective conductivity for the model for S12 is estimated as

$$
\left(\frac{\sigma_{\text {eff,S12 }}}{\sigma_{\text {bulk }}}\right)=0.4976 \pm 0.0034
$$

where the errors indicate the $\approx 95 \%$ confidence interval of the average given by the sample mean $\pm 2 \frac{\mathrm{SD}}{\sqrt{n}}$, where both quantities were estimated form the 20 realizations with edge length 512 .

The corresponding study for the sample S14 is shown in Figure 14. Due to the larger structure size, the RVE for S14 is slightly larger, yet for an edge length for about 384 voxel corresponding to a physical volume with edge length $\approx 1,9 \mu \mathrm{m}$, the edge effects are negligible and the relative precision on 20 realizations is about $\pm 2 \%$. 


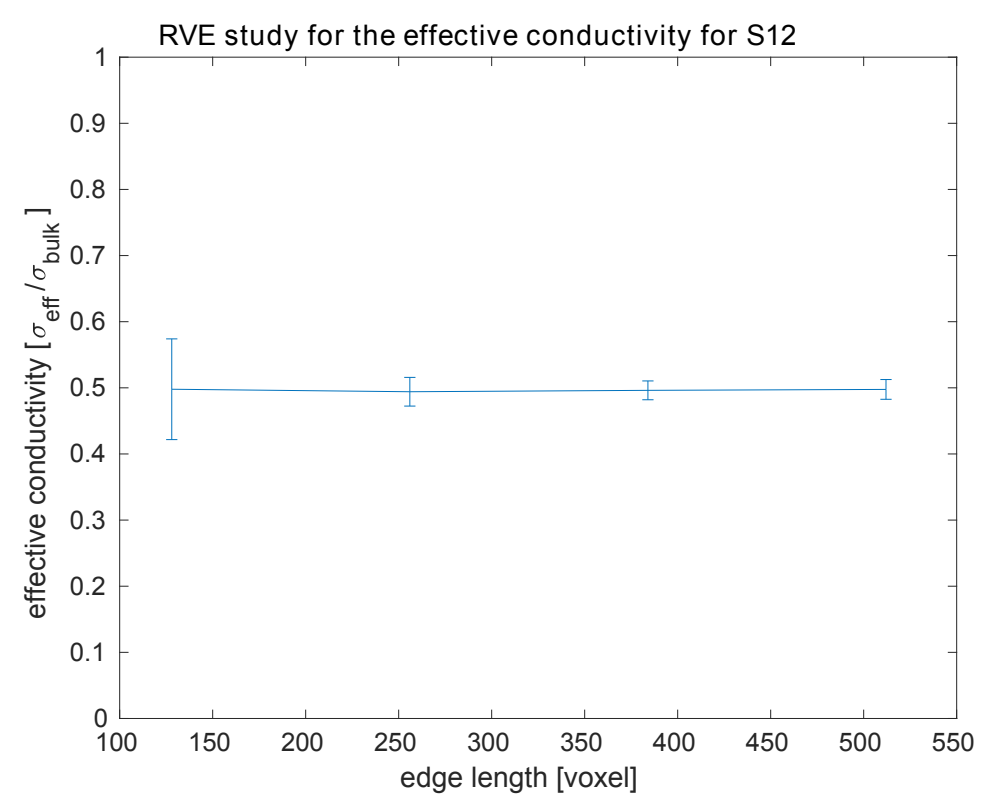

Fig. 13 Study on the RVE of the conductivity the model with parameters for S12. The error bars corresponding to the $95 \%$ confidence interval for the individual realizations.

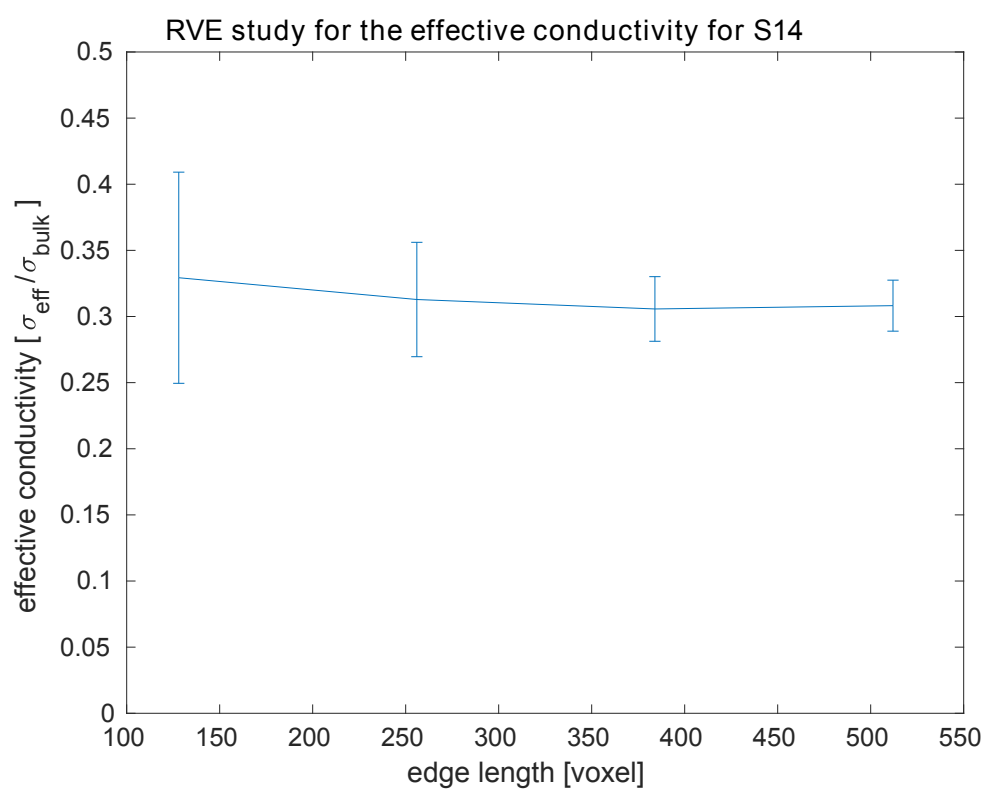

Fig. 14 Study on the RVE of the conductivity the model with parameters for S14. The error bars corresponding to the $95 \%$ confidence interval for the individual realizations. 
The average effective conductivity for S14 estimated from a volume of $512^{3}$ voxels is

$$
\left(\frac{\sigma_{\text {eff }, \mathrm{S} 14}}{\sigma_{\mathrm{bulk}}}\right)=0.308 \pm 0.0044
$$

With the error again being the $\approx 95 \%$ confidence interval of the average.

The estimated conductivities of the two samples are much lower than their upper Wiener and Hashin-Shtrikman bounds (Table 5), which would provide poor estimates. Third-order bounds provide better estimates, but still with an overestimation of the conductivity.

\subsubsection{Effective Diffusivity}

The other important transport property considered in this work is the effective diffusivity in the pore space. Since the charge carriers in the electrolyte are transported by diffusion in the electrolyte, which in turn is filling the pore space, the effective diffusivity has a major impact on the charging speed of the EDLC electrode. The effective diffusivity is estimated using the same procedure as for the effective conductivity. In the model, it was assumed, that the solid phase is impregnable for charge carriers and hence the diffusivity of the solid phase vanishes. The diffusivity of the pore space was set to one. RVE studies for the effective diffusivity for both samples are shown in Fig. 15 and 16. Both studies show, that for edge lengths of the computational domain of more than 256 Voxels, edge effects essentially vanish and the relative precision reduces to less than $\pm 1 \%$. This corresponds to a representative volume with an edge length of about $l \approx 0.6 \mu \mathrm{m}$ for $\mathrm{S} 12$ and $l \approx 1.2 \mu \mathrm{m}$ for $\mathrm{S} 14$, respectively.

For the parameter set representing the model for S12, the effective diffusivity was estimated from 20 realization with edge length of 512 Voxels to

$$
\left(\frac{D_{\text {eff }, \mathrm{S} 12}}{D_{\text {bulk }}}\right)=0.097 \pm 0.0048
$$

For the parameter set representing S14 the effective diffusivity was estimated to

$$
\left(\frac{D_{\text {eff,S14 }}}{D_{\text {bulk }}}\right)=0.24 \pm 0.0042
$$

In both cases, the errors represent the $\approx 95 \%$ confidence interval of the average, computed as for the effective conductivity.

Comparison to the bounds in Table 6 shows that the simulated values are closest to the BSM bounds.

Additionally, the diffusivities of the samples have been measured by electroimpedance spectroscopy resulting in the values in Table 7. A comparison shows that the qualitative behavior of the two samples is reproduced, yet quantitatively the level of agreement is low. 


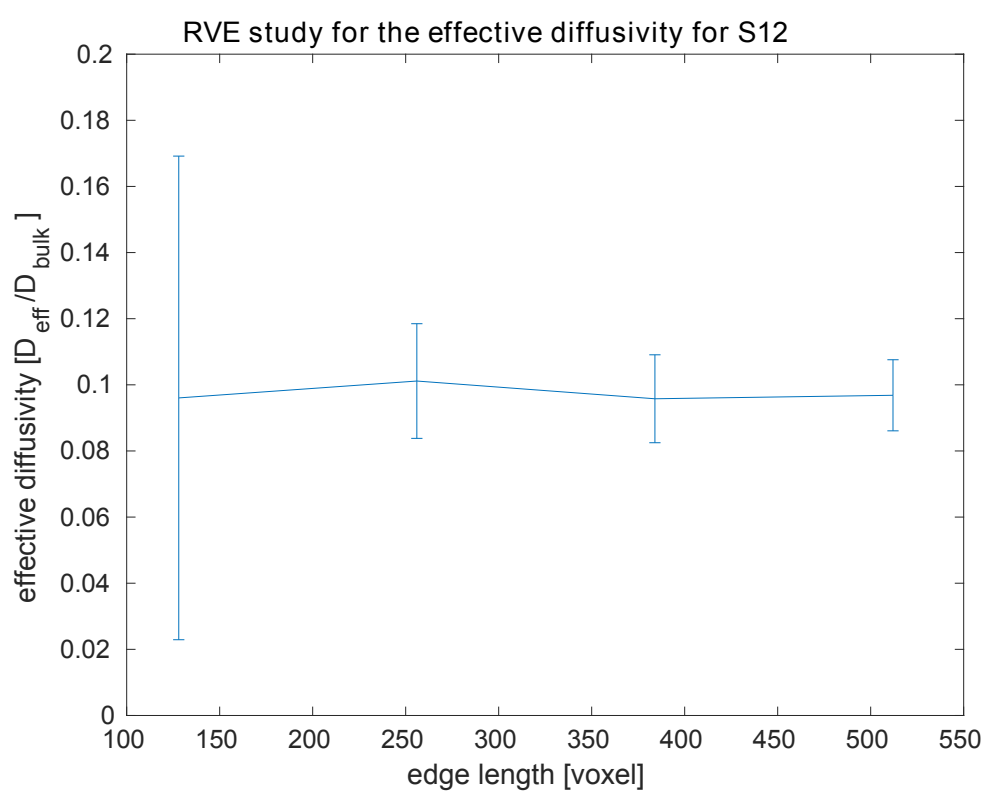

Fig. 15 Study on the RVE of the effective diffusivity of the model with parameters for S12. The error bars corresponding to the $95 \%$ confidence interval for the individual realizations.

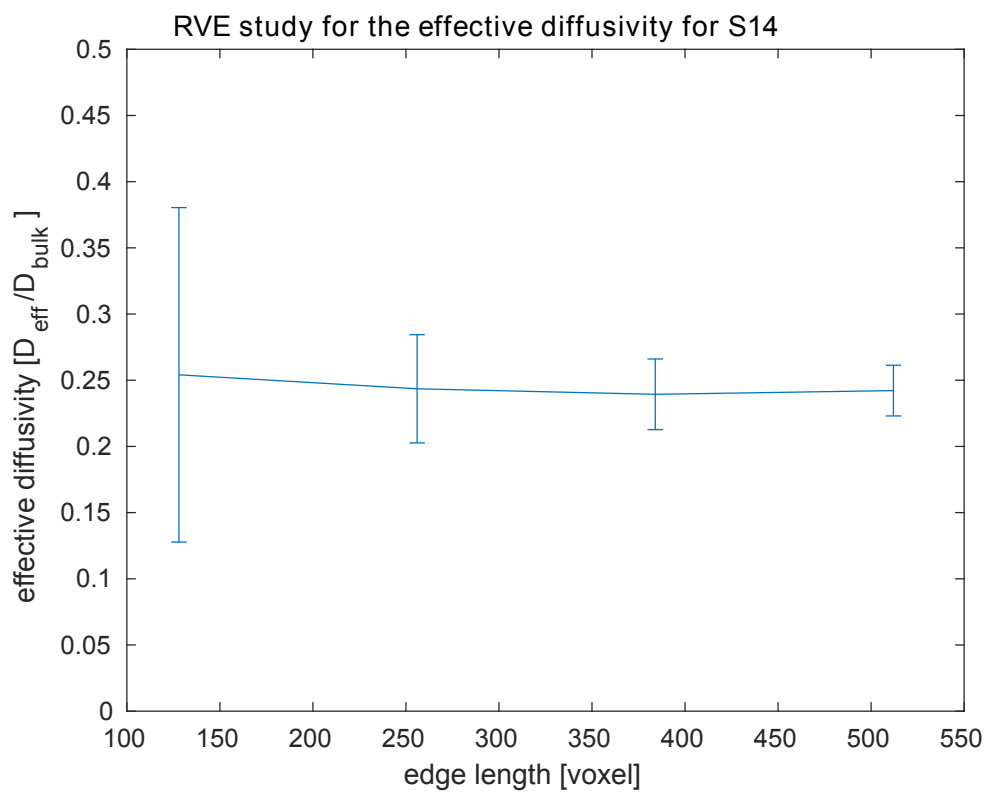

Fig. 16 Study on the RVE of the effective diffusivity of the model with parameters for S14. The error bars corresponding to the $95 \%$ confidence interval for the individual realizations. 
Table 7 Measured and simulated diffusivities of the two samples, assuming a bulk diffusivity of $D_{\text {bulk }}=1.08 \times 10^{-6} \frac{\mathrm{cm}^{2}}{\mathrm{~s}}$.

\begin{tabular}{lcc}
\hline Sample & $D_{\operatorname{Exp}}$ & $D_{\mathrm{Sim}}$ \\
\hline $\mathrm{S} 12$ & $1.07 \times 10^{-6} \frac{\mathrm{cm}^{2}}{\mathrm{~s}}$ & $0.097 \frac{\mathrm{cm}^{2}}{\mathrm{~s}_{2}}$ \\
$\mathrm{~S} 14$ & $4.89 \times 10^{-6} \frac{\mathrm{cm}^{2}}{\mathrm{~s}}$ & $0.24 \frac{\mathrm{cm}^{2}}{\mathrm{~s}}$ \\
\hline
\end{tabular}

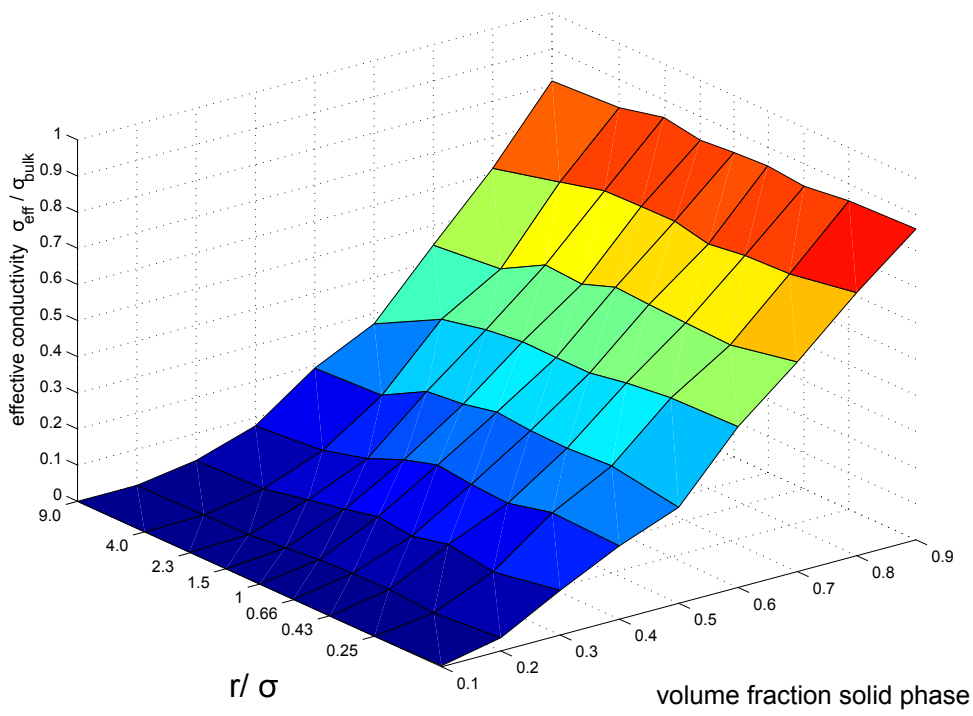

Fig. 17 Parameter study of the effective conductivity of the model described in Section 4.2.

\section{Parametric Study}

Finally, a parametric study was performed, to assess the impact of the different model parameters on the effective conductivity and diffusivity of the resulting microstructures. To this end, the realizations of the model were generated with pore volume fractions ranging from $10 \%$ to $90 \%$. Due to the linear nature of the conductivity problem, the absolute size of the radii and filter masks do not affect the effective properties. Thus, we limited the study to the estimation of the influence of the ratio between the radii of the spheres of the Boolean model and the size of the filter mask. The resulting effective conductivity and diffusivity are shown in Fig. 17 and 18.

As can be seen, the ratio between the initial sphere radius and size of the filter mask has only a minute influence on the effective conductivity. Hence, it should in most cases be sufficient, to fit a Boolean model of spheres to the microstructure and estimate the effective conductivity and diffusivity on its realizations. 


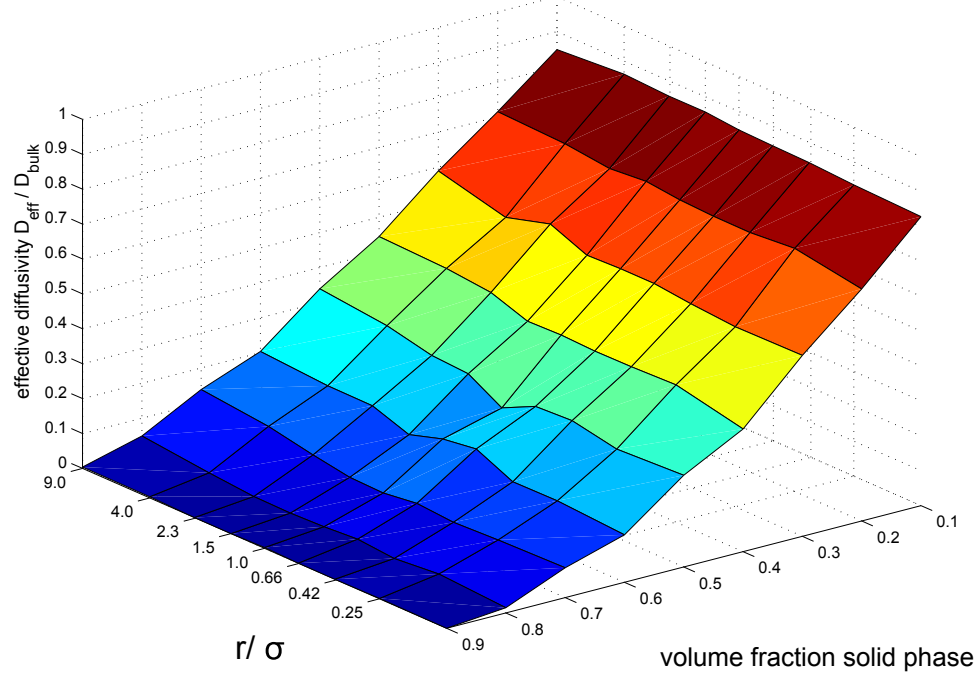

Fig. 18 Parameter study of the effective diffusivity of the model described in Section 4.2.

\section{Comparison with Experiment}

Experimentally, the gravimetric capacitance for both materials were measured using electroimpedance-spectroscopy. The corresponding capacitance values for both materials are:

$$
\begin{aligned}
& C_{G, S 12}=157 \frac{F}{g}, \\
& C_{G, S 14}=113 \frac{F}{g} .
\end{aligned}
$$

To compare the measurements with the calculated values, we calculate the ratio of the volumetric capacitances of the two samples through

$$
\begin{aligned}
& C_{V, S 12}=C_{G, S 12} p_{S 12} \rho, \\
& C_{V, S 14}=C_{G, S 14} p_{S 14} \rho,
\end{aligned}
$$

where $p_{S 12}$ and $p_{S 14}$ are the volume fractions and $\rho$ is the density of the microporous phase of the two samples in $\frac{g}{\mathrm{~cm}^{3}}$.

Since, the density of the microporous phase $(\rho)$ is not known, only the ratio of the volumetric capacitances of the two materials can be compared. The ratio of the measured volumetric capacitances is given by

$$
\frac{C_{V, S 12}}{C_{V, S 14}}=\frac{C_{G, S 12} p_{S 12} \rho}{C_{G, S 12} p_{S 14} \rho}=1.79,
$$

where the density $\rho$ cancels out. By comparison, the ratio between the capacitances of the modeled structures is

$$
\frac{p_{S 12}}{p_{S 14}}=1.288
$$


Table 8 Specific surface of the samples measured on the segmented images $a_{\text {seg }}$ as well as on the modeled microstructures $a_{\text {mod }}$.

\begin{tabular}{lcc}
\hline Sample & $a_{\text {seg }}$ & $a_{\text {mod }}$ \\
\hline $\mathrm{S} 12$ & $3.1 \times 10^{5} \frac{\mathrm{cm}^{2}}{\mathrm{~cm}^{3}}$ & $3.18 \times 10^{5} \frac{\mathrm{cm}^{2}}{\mathrm{~cm}^{3}}$ \\
$\mathrm{~S} 14$ & $7.1 \times 10^{4} \frac{\mathrm{cm}^{3}}{\mathrm{~cm}^{3}}$ & $6.02 \times 10^{4} \frac{\mathrm{cm}^{3}}{\mathrm{~cm}^{3}}$ \\
\hline
\end{tabular}

If the charge was stored only on the micropore surface, these ratios should be the same. Possible reasons for this discrepancy are that a double layer is forming on the mesopore surface, which has not been considered in the simulation. Also, the bulk volumetric capacitances of the solid phase might differ between the two samples, due to the possible presence of micropores with different volume fractions, unresolved by the FIB-SEM images. Since the sample S12 has a larger measured capacitance relative to S14 and also a larger surface area of the mesopores, which would explain a larger discrepancy if a double layer would be formed on the mesopore surface, the first possibility is further explored in this study.

\section{Prediction of the Volumetric Capacitance Including a Double Layer on the Mesopore Surface}

It is assumed, that the total volumetric capacitance is a linear combination of the volumetric capacitance and the areal capacitance of the mesopore double layer multiplied by the specific surface area of the mesopores

$$
\begin{aligned}
& C_{V, S 12}=p_{S 12} C_{V}+a_{S 12} C_{A}, \\
& C_{V, S 14}=p_{S 14} C_{V}+a_{S 14} C_{A} .
\end{aligned}
$$

The specific surface areas were measured on the segmented images as well as on the modeled microstructures. The resulting values are given in table 8 .

Since the volumetric capacitances are known, only up to a factor $\rho$, Eqs. 31, 28 and 29 can be solved for the ratio of bulk volumetric capacitance coming from the micropores and volumetric surface capacitance coming from the mesopores. Using the measured surface area of the modeled microstructures, this leads to the following areal capacitance and volumetric capacitances depending on $\rho$

$$
\begin{aligned}
C_{V} & =99.3 \rho \frac{F}{g}, \\
C_{A} & =1.12 * 10^{-4} \rho \frac{F c m}{g} .
\end{aligned}
$$

For the sample S12 this yields the following contributions to the capacitance:

$$
\begin{aligned}
& C_{V, S 12}=C_{V} p_{S 12}=66.6 \rho, \\
& C_{V, S 12}^{A}=C_{A} a_{S 12}=38.6 \rho .
\end{aligned}
$$

Hence, in the sample S12 with a total capacitance of $C_{S} 12=105.9 \rho \frac{F}{\mathrm{~cm}^{3}}, 36 \%$ of the total volumetric capacitance arises from the double layer on the mesopore 


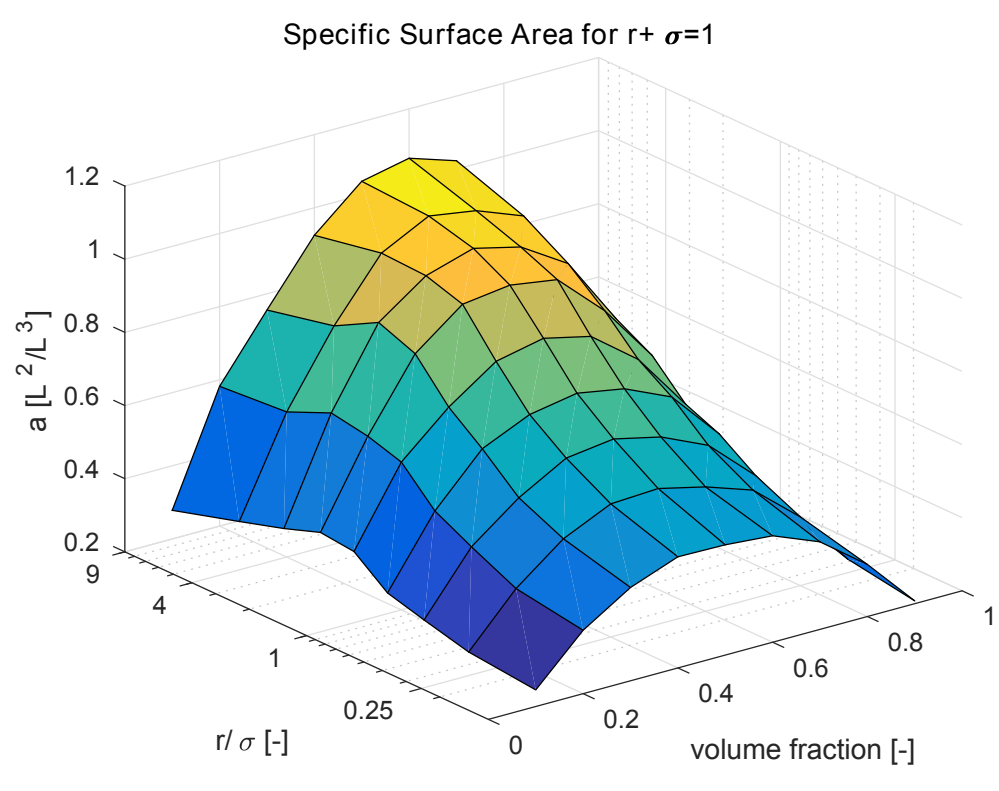

Fig. 19 Parameter study of the effective surface area of the model described in Section 4.2 with spheres of radius one.

surface and the remaining $66.6 \%$ from the micropores. For the sample S14 the corresponding relations are:

$$
\begin{aligned}
& C_{V, S 12}=C_{V} p_{S 12}=51.7 \rho, \\
& C_{V, S 12}^{A}=C_{A} a_{S 12}=7.09 \rho .
\end{aligned}
$$

Hence, in the sample S14, $12 \%$ of the capacitance of $C_{S} 14=58.76 \rho \frac{F}{\mathrm{~cm}^{3}}$ comes from the mesopores and $87.9 \%$ comes from the microspores.

In order to estimate the optimal properties of the mesopore morphology, a parameter study was done to estimate the dependence of specific surface area on the model parameters. For the parameter study ten realizations with dimension $256 \times 256 \times 256$ voxels were generated of the model for the same parameter values as in Sect. 6, i.e. with $r+\sigma=30$ voxels. Then the surface area has been rescaled for a model with unit spheres of radius one, i.e. the expected surface area within a unit cube. The resulting specific surface areas are shown in Fig. 19.

For physical structures, the specific surface area for the radius $r$ can be derived by the scaling law:

$$
A(r)=\frac{A(r=1)}{r}
$$

Hence, for a sphere radius of $20 \mathrm{~nm}$ the maximal specific surface area is

$$
S_{A} \approx \frac{1 \frac{\text { voxel }^{2}}{\text { voxel }^{3}}}{20 * 10^{-9} \mathrm{~m}}=0.05 * 10^{9} \frac{\mathrm{m}^{2}}{\mathrm{~m}^{3}}=5 * 10^{5} \frac{\mathrm{cm}^{2}}{\mathrm{~cm}^{3}}
$$

To improve the microstructural properties using the model, we use Eqs. 31, 32, 37 and the computed data in Fig. 19 for a ratio of $\frac{r}{\sigma}=1.5$, which is close to the 


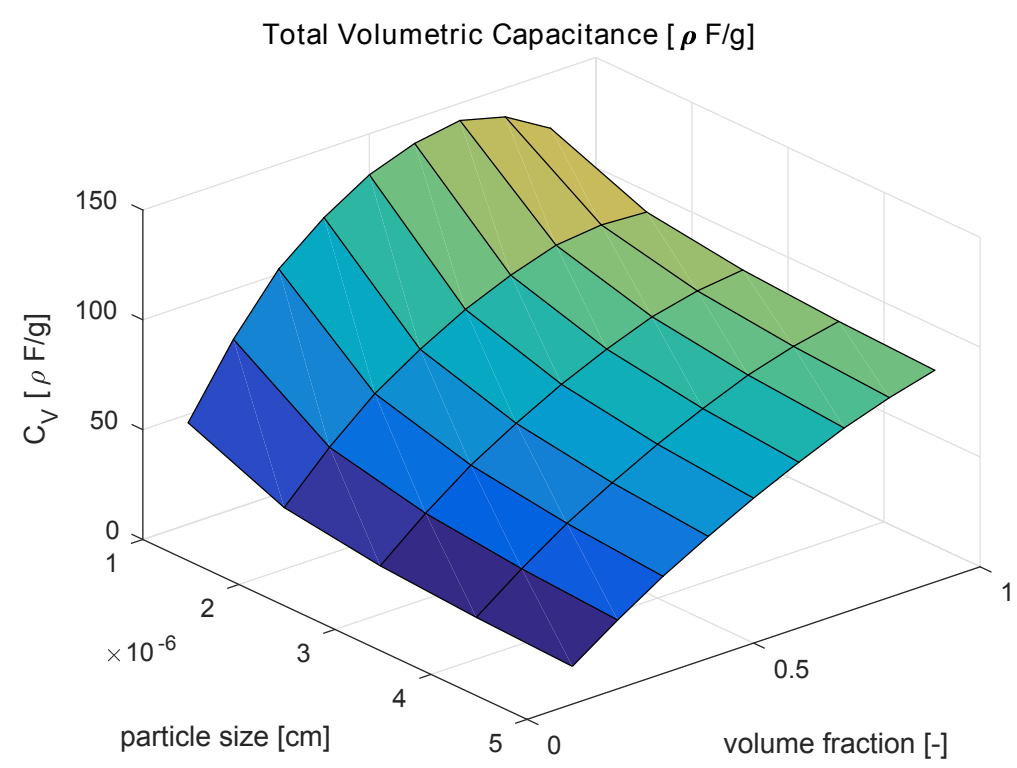

Fig. 20 Parameter study of the total capacity of a structure of the model described in Sect. 4.2, with spheres of radius $d$, the volumetric and areal capacities from Eq. 32 and $\frac{r}{\sigma}=1.5$.

fitted value for both microstructures of $\frac{r}{\sigma} \approx 1.4$. We then compute the volumetric capacitance depending on $d=r+\sigma$ between $10 \mathrm{~nm}$ and 50nm and the volume fraction $p$ between 0.1 and 0.9 . The resulting capacitances are shown in Fig. 20.

As can be seen, the largest capacitance is reached for the smallest radius, i.e., the smallest structure size of the model. This is due to the scaling in Eq. 37. For larger structures, the specific surface area is small, meaning that the volumetric term is dominant. Hence in the large structure regime, the maximal capacitance is computed for the largest volume fraction $p$. Yet, for smaller structures, the surface term dominates. This means, that the maximal capacitance is reached for a maximal surface area.

As shown in Fig. 19, the maximal surface area is reached for a volume fraction of $p=0.6$ and minimal to no smoothing. For these parameters, the specific surface area peaks at around $a\left(p=0.6, \frac{r}{\sigma} \rightarrow \infty\right) \approx 1$.

\section{Conclusion}

In this paper, nanoporous materials for EDLC electrodes are investigated using FIB-SEM Nanotomography imaging, combined with automatic segmentation and random set modeling. It could be shown that transport properties could be predicted combining the structural model with simulation using the FFT method. The results agree qualitatively with experimental measurements. Furthermore, it could be shown that the capacitance values can be predicted when combining the mentioned techniques modeling based on the geometrical characteristics of the model. In this case, the experimental values do coincide very well with the experimental 
results. Finally, by varying the parameters of the structural model, improvements to the microstructure could be suggested. In conclusion, it was shown that by combining techniques of microimaging, image processing, stochastic modeling and simulations, material properties of EDLC electrodes could be predicted theoretically.

\section{Outlook}

The research presented in this paper focuses on the context of energy storage in EDLC's but can similarly be applied to lithium ion batteries or other nanoporous materials. In both cases, batteries as well as EDLC's the electrodes comprise mostly of a micro- or nanoporous carbon structure filled with an electrolyte. Furthermore, the techniques demonstrated here can be amended and improved individually. As the FIB technology improves, the image quality of finer structures like the ones investigated here can be improved, yielding a better segmentation. Also, since automatic segmentation for FIB-SEM images is a relatively new field, improved algorithms can improve the accuracy of the segmentation. Concerning the structural modeling, replacing the Boolean model, which serves as a basis for the modified model by a cherry-pit model, can improve the modeling of the sphere packing. Finally, improved physical simulations as mentioned in the introduction can be used to further enhance the prediction of the capacitance.

Acknowledgements This work was partly funded by the German Federal Ministry of Education and Research, project AMiNa (03MS603D). The authors are also grateful for the support of DFDK-CDFA (grant CDFA-02-11).

\section{References}

Balach J, Miguel F, Soldera F, Acevedo D, Mücklich F, Barbero C (2012) A direct and quantitative image of the internal nanostructure of nonordered porous monolithic carbon using fib nanotomography. Journal of Microscopy

Balach J, Soldera F, Acevedo DF, Mücklich F, Barbero CA (2013) A direct and quantitative three-dimensional reconstruction of the internal structure of disordered mesoporous carbon with tailored pore size. Microscopy and Microanalysis 19:745-750

Barsoukov E, Macdonald J (2005) Impedance Spectroscopy: Theory, Experiment, and Applications. Wiley, London

Bazant MZ, Thornton K, Ajdari A (2004) Diffuse-charge dynamics in electrochemical systems. Phys Rev E 70:021,506

Beran M (1965) Use of the variational approach to determine bounds for the effective permittivity in random media. Il Nuovo Cimento Series 10 38(2):771782

Conway B (2013) Electrochemical Supercapacitors: Scientific Fundamentals and Technological Applications. Springer US

de Levie R (1963) On porous electrodes in electrolyte solutions: I. capacitance effects. Electrochimica Acta 8(10):751 - 780 
Duffy D (2001) Green's Functions with Applications. Applied Mathematics, CRC Press, Boca Raton

Eyre DJ, Milton GW (1999) A fast numerical scheme for computing the response of composites using grid refinement. The European Physical Journal Applied Physics 6:41-47

Grzhibovskis R, Heintz A (2005) A convolution-thresholding approximation of generalized curvature flows. SIAM Journal on Numerical Analysis 42(6):26522670

Hutzenlaub T, Becker J, Zengerle R, Thiele S (2013) How coarsening the 3d reconstruction of a porous material influences diffusivity and conductivity values. ECS Electrochemistry Letters 2(2):F14-F17

Jeulin D (2000) Random texture models for material structures. Statistics and Computing 10(2):121-132

Jeulin D (2005) Random structures in physics. In: Bilodeau M, Meyer F, Schmitt M (eds) Space, Structure and Randomness, Lecture Notes in Statistics, vol 183, Springer New York, pp 183-219

Jeulin D, Moreaud M (2005) Multi-scale simulation of random spheres aggregatesapplication to nanocomposites. In: 9th European Congress on Stereology and Image Analysis, Zakopane, Poland, vol 1, pp 341-348

Jørgensen P, Hansen K, Larsen R, Bowen J (2010) A framework for automatic segmentation in three dimensions of microstructural tomography data. Ultramicroscopy $110(3): 216-228$

Kanit T, Forest S, Galliet I, Mounoury V, Jeulin D (2003) Determination of the size of the representative volume element for random composites: statistical and numerical approach. International Journal of Solids and Structures 40(1314):3647 $-3679$

Matheron G (1967) Elements pour une théorie des milieux poreux. Masson, Paris

Matheron G (1975) Random Sets and Integral Geometry. Wiley series in probability and mathematical statistics. Probability and mathematical statistics, John Wiley, London

Milton G (1982) Bounds on the elastic and transport properties of two-component composites. Journal of the Mechanics and Physics of Solids 30(3):177 - 191

Nanjundappa A, Alavijeh AS, Hannach ME, Harvey D, Kjeang E (2013) A customized framework for 3-d morphological characterization of microporous layers. Electrochimica Acta 110:349 - 357

Paasch G, Micka K, Gersdorf P (1993) Theory of the electrochemical impedance of macrohomogeneous porous electrodes. Electrochimica Acta 38(18):2653 - 2662

Papanicolau G, Bensoussan A, Lions J (1978) Asymptotic Analysis for Periodic Structures. Studies in Mathematics and its Applications, Elsevier Science, Amsterdam

Prill T, Schladitz K, Jeulin D, Faessel M, Wieser C (2013) Morphological segmentation of FIB-SEM data of highly porous media. Journal of Microscopy 250(2):77-87

Roßberg K, Paasch G, Dunsch L, Ludwig S (1998) The influence of porosity and the nature of the charge storage capacitance on the impedance behaviour of electropolymerized polyaniline films. Journal of Electroanalytical Chemistry 443(1):49 - 62

Salzer M, Spettl A, Stenzel O, Smått JH, Lindén M, Manke I, Schmidt V (2012) A two-stage approach to the segmentation of FIB-SEM images of highly porous 
materials. Materials Characterization 69(0):115 - 126

Sánchez-Palencia E (1980) Non-homogeneous media and vibration theory. Lecture notes in physics, Springer-Verlag

Serra J (1982) Image Analysis and Mathematical Morphology. Academic Press, London

Spall JC (1992) Multivariate stochastic approximation using a simultaneous perturbation gradient approximation. IEEE Transactions on Automatic Control 37:332-341

Torquato S, Stell G (1985) Bounds on the effective thermal conductivity of a dispersion of fully penetrable spheres. International Journal of Engineering Science $23(3): 375-383$

Wang H, Pilon L (2012) Physical interpretation of cyclic voltammetry for measuring electric double layer capacitances. Electrochimica Acta 64:130 - 139

Willot F, Abdallah B, Pellegrini YP (2014) Fourier-based schemes with modified green operator for computing the electrical response of heterogeneous media with accurate local fields. International Journal for Numerical Methods in Engineering 98(7):518-533 